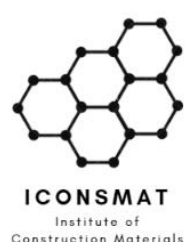

Institute of
Construction Matorials
Content list available at ICONSMAT

Journal of Construction Materials

Journal homepage: www.iconsmat.com.au/publication
Article history:

Received 20 February 2020

Received in revised form

10 March 2020

Accepted 21 March 2020

Available online

2 April 2020

\title{
Automatic sorting of recycled aggregate using image processing and object detection
}

\author{
Farid Sartipi*1 \\ ${ }^{1}$ Institute of Construction Materials, NSW, Australia \\ *Corresponding author: Farid Sartipi, Associate Researcher, Institute of Construction Materials, NSW, 2747, Australia. \\ E: farid.sartipi@iconsmat.com.au P: (+61) 0416731647
}

\begin{abstract}
With the growth of population and expansion of cities, the use of recycled construction materials has attracted builder's attention. Governments also initiate the utilization of environmental practices through legislation, regulations, and development controls. Despite all the efforts so far, the use of recycled aggregate is still not as popular as it should mainly due to a few mechanical deficiencies encountered. In this study, the consistency of the particles had gone subject to investigation, i.e. sorting various components found in recycled aggregate including brick, ceramic, natural aggregate, glass, plastic, etc. An image processing apparatus in companion with computer codes capable of object detection had been assembled and tested. Future studies can lead to the development of fully integrated robotic module for sorting recycled aggregate using robotic arms.
\end{abstract}

DOI: 10.36756/JCM.v1.3.3 @2020 Institute of Construction Materials

\section{Keywords}

Recycled Aggregate; Image processing; Object detection 


\section{Introduction}

Humans seeking for a more dynamic life style, in the pursuit of the so called "modern life style" (whether right or wrong as a decision), move to large cities. Wherever population gathers for a long period of time maintenance and the renovation of old buildings and infrastructures often takes a large chunk of the government budget. Structures that no longer are capable of addressing the needs of the modern human will eventually go under demolition. Waste treatment as the following step, in the last couple of decades was subject to the questions of researchers and the scientific community e.g. how to recycle the waste material in the new construction? What are the solutions to enhance the properties of the recycled concrete? Is it possible to chemically strengthen the properties? Where do the automated robots stand in this process?

This is where the concerns about the sustainable construction development had arisen. Many methods had been proposed since decades ago including comprehensive life cycle assessment[1-6], use of recycled aggregate in concrete[7-12], implementing regulatory policies and procedures, carbon tax[13, 14], $\mathrm{CO}_{2}$ capture[11, 15-18], use of carbon particles in concrete[19, 20], etc.

Depending on the type of the recycling plant, the steps undertaken to produce a usable recycled aggregate varies. Nonetheless, regardless of the chronological order, the process contains crushing, sorting, washing, and piling. Crushing, affects the size of the aggregates and is actually the step in which determines the supply of appropriate product to the user's demand. Washing, although optional in some recycling plants, increases the quality of the final product as throughout this process dust and other fine particles are being filtered. Piling is important specially in humid areas in terms of the water content of the aggregates. Sorting, the main topic of this article had remain a main challenge for decades until the last few years where robotic advancements had come into assist. The main reason as for why recycling plants seek to separate the components is the ability for predicting the quality of concrete made with these aggregates. In fact, the portion of recycled aggregate varies significantly from case to case and location by location. This leaves the users, concrete contractors and batching plants with a lot of uncertainties in regards to the standardization of recycled aggregate. What can be determined as obvious is the fact that a sample of recycled aggregate with a, for instance, portion of say 30\% brick, 30\% natural aggregate, 30\% ceramic, etc. performs different properties compared with a sample portion of say $25 \%$ brick, $35 \%$ natural aggregate, $20 \%$ ceramic, $10 \%$ glass, etc.

Automatic sorting is basically aims to bring a solution for the heterogeneity issues with recycled aggregate. The first step in this context is identification. In the following comes dimension recognition, data storage, and processing. Few identification methods had been studies so far including hyperspectral imaging, and the use of 3D cameras[21, 22]. The pros and cons of each of these individual methods are shown in the following table 1 : 
Type of Camera Advantage

Disadvantage
Accurate identification of particle type

(Brick, ceramic, etc)
- Unable to distinguish the dimensions

- $\quad$ Expensive

- Unable to identify the particle type

- Expensive

The above table indicates that if a proper automatic sorting system is ought to be developed a combination of two types of cameras are required in order to form the identification matrix which will be used further in the processing step. Furthermore, the combination of two different camera outputs is a challenge by itself. An operable module equipped with hyperspectral and 3D imaging cameras in only a small scale for laboratory purposes costs about AUD $\$ 10,000$. Of course the price for the assembly of industrial system cost even more which makes the investment opportunities low.

Here a cost effective image processing protocol has been developed based on colour coding of recycled aggregate. The system is also capable of recognizing the geometric dimensions of the particles which facilitates the processing steps in the following stages.

\section{System Setup}

The camera used in this project contains a built-in micro-controller for object detection which facilitates the computer programming processes. It uses colour-based object detection protocol which in the case of recycled aggregate is so useful as the particles are mostly distinguishable from their colour i.e. brick is usually red in colour, blue metal has dark blue colour code, etc. Prior to the start of the sorting process and depending on the source of the demolition waste, the camera needs to be trained in advanced. In simple words, the camera needs to be set manually in separate steps to differentiate between, for example, brick and blue metal. The system processes a captured image 60 times per second. 7 Distinct colours had been coded in the system. This means that a batch of recycled aggregate can contain up to 7 different materials. The software is compatible with Windows devices.

The geometrical dimensions of the particles are being processed and stored using a separate microcontroller. One of the key advantageous of this module compared with the ones developed in the other studies is the utilization of one camera solely. The data processing and the CPU requirements are considerably condensed in this method which brings a competitive advantage in terms of time consumed for sorting process. In other words, the users are capable of producing a larger amount of heterogenic recycled aggregate per unit of time once this device is used. Figure 1 demonstrates the system instalment. 


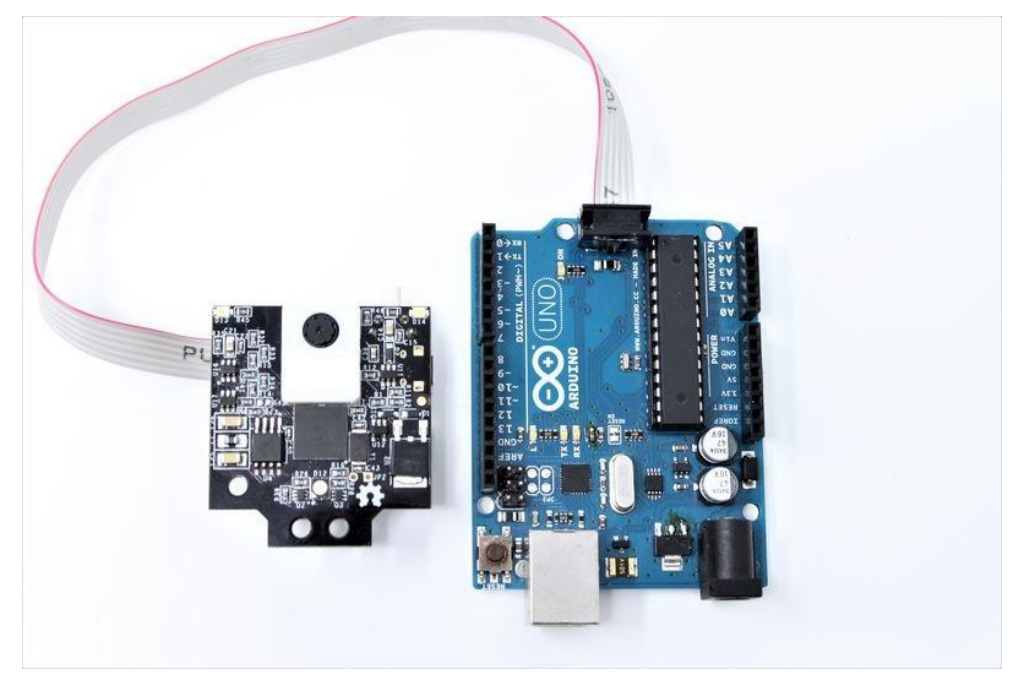

Figure 1 Left: The camera for colour-based object detection; Right: Microcontroller for geometrical dimension recognition

From the software point of view, the camera loads the following script on the computer for object detection. The speed of the serial port needs to be set high because of the high volume of data being received from the setup. The program first identifies the blocks and counts the number of blocks subsequently using a FOR loop.

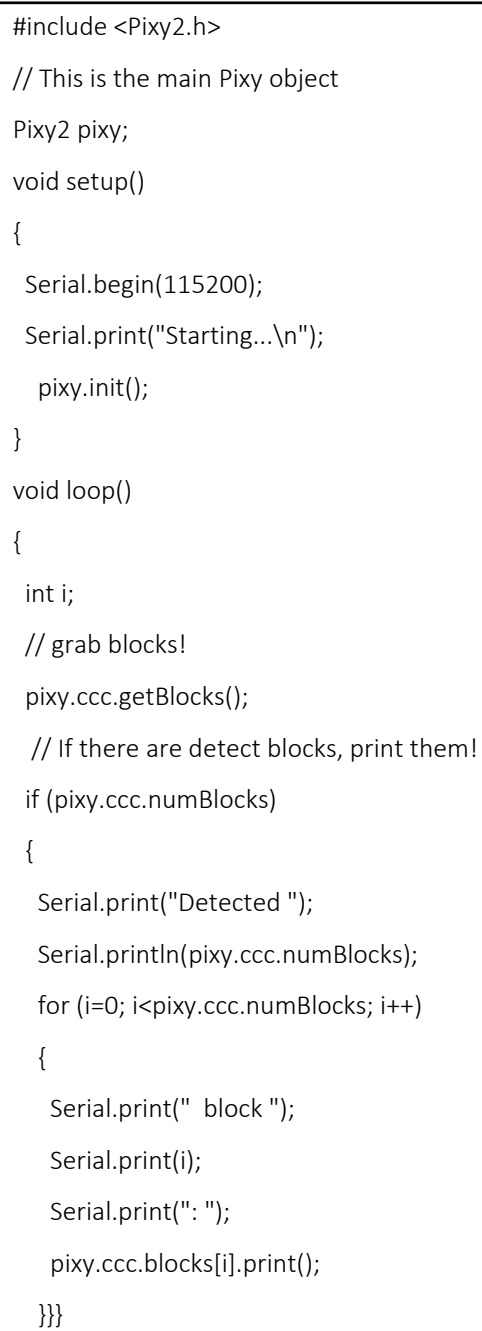




\section{Discussion}

The accuracy of the system plays a crucial role in the successful commercialization process. In other words a need exist for the accuracy of the number of blocks identified and the size of the block reported by the machine. An array of 8 elements/attributes had been produced each $1 / 60$ of a second including the attributes such as the signature of the block (assigned particle name e.g. brick, blue metal, plastic, etc), $x$ and y coordination of the block in the frame, width and height of the block, index number and age (which outputs the time-dependent sequence when the block appeared in the frame for the first time). A rough analysis on the output data had revealed that with a minor adjustment on the camera settings, the accuracy can be enhanced to up to $87.5 \%$. This largely depends on the quality of the recycled materials received as different batches may contain a variety of materials with different colours. One of key important factors in the system adjustment is the colour code of the background. In case one decides to install the presented image processing system on a conveyor belt, the colour of the conveyor belt affects the identification process.

Assigning signatures to the particles is one of the important aspects of this system. It requires a professional operator to calibrate the machine in an utmost efficient way in order to maximize the accuracy of the system in identifying the nature of the particles whether would be brick, blue metal, plastic or others. A manual control system is available exactly for this purpose. Since the system runs on RGB (Red, Green, Blue) processing, the intensity of each colour can be determined manually. In other words, the recycling batch needs a preassessment by the operator to distinguish the colour codes. For more accuracy, it is recommended to have a white LED installed beside the camera shedding light for more colour contrasting. Below an example of an unsuccessful object detection is presented in figure 2 . The output data presented in figure 3 relates to a highly accurate processing where 7 objects out of a total 8 had been detected. The attributes recorded are presented in the same figure as well.

From the managerial perspective, an automatic sorting system as such is capable of enhancing the physical/mechanical properties of recycled concrete while the final unit price of the recycled aggregate remains low. Concrete contractors, once the system had been utilized widely, shift to the use of recycled aggregate eagerly as opposed to the current hesitative preference of using natural aggregate. One can argue the economic impact of having such system operating in the market. Of course it leads to loss of a few jobs as robotics often replaces old fashioned jobs with new ones. Another economic aspect is the change in the supply and demand of recycled and natural aggregate. Once the eagerness to consume a higher quantity of recycled aggregate the demand for natural aggregate drops. Subsequently, as a positive result, the exploitation of natural resources and its terrifying consequences will be limited[23-25]. 


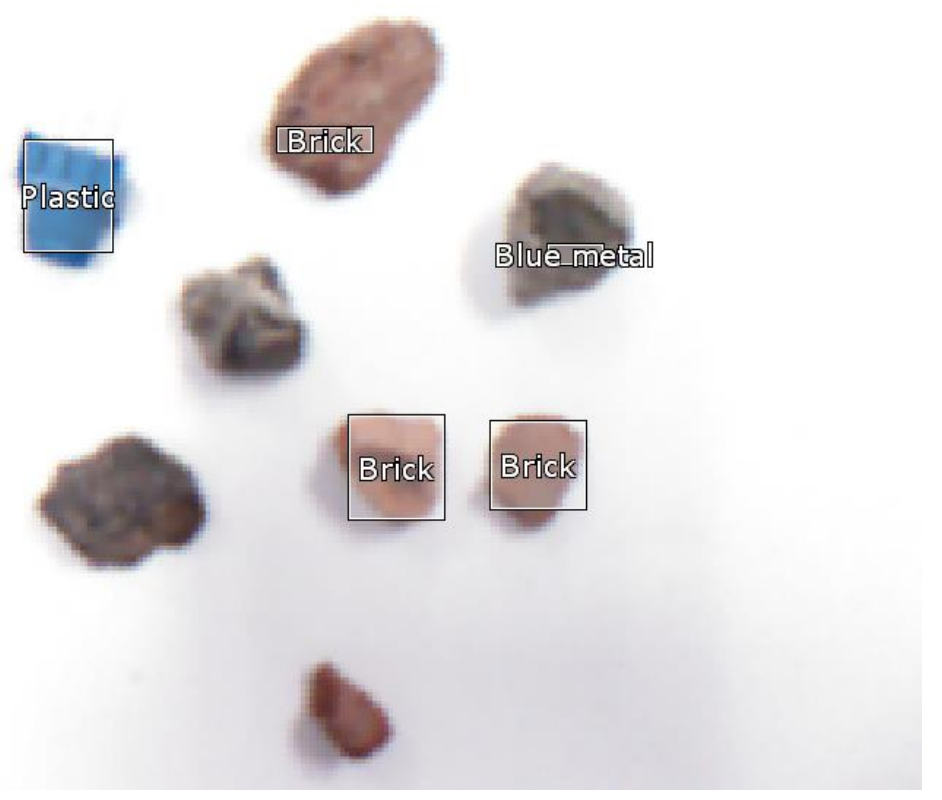

Figure 2 An example of a poor detection; the process can be optimized by adjusting the color codes and the background brightness for a more distinguishable contrast

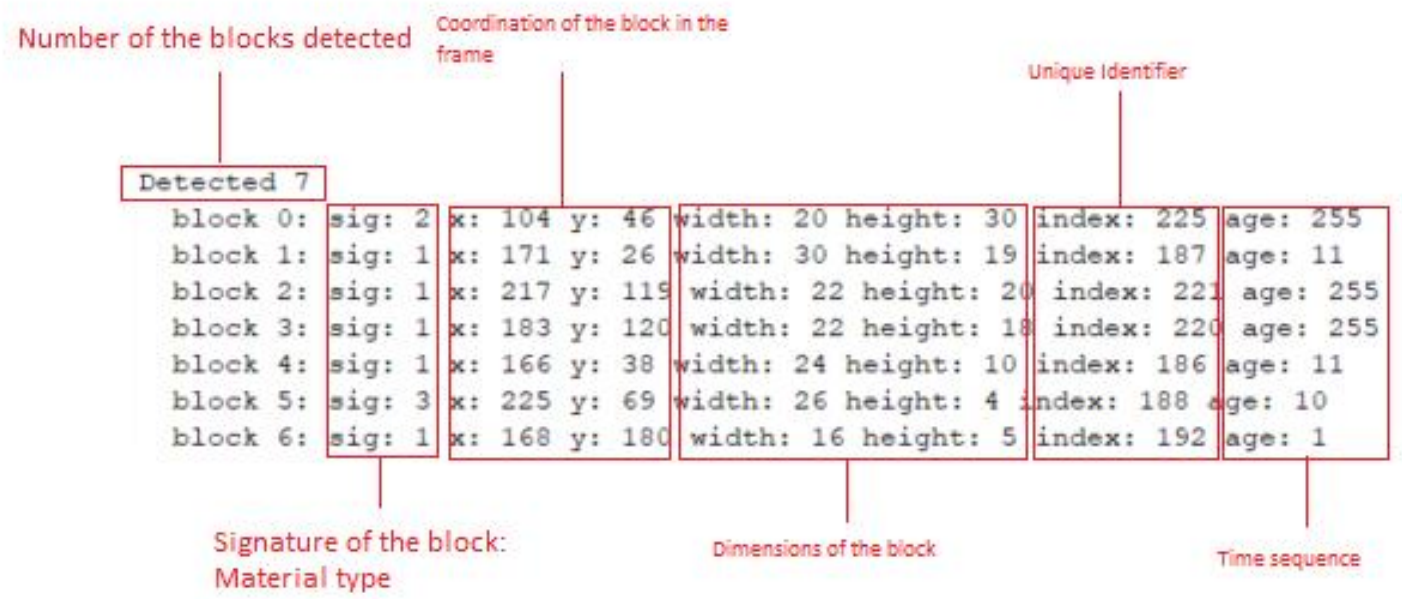

Figure 3 Output data from an example of a successful detection. 7 item out of 8 had been correctly identified and listed 


\section{Conclusion}

Construction and demolition waste recycling plants are seeking for a more automated sorting procedure in order to tackle the drawbacks expressed by the concrete batching plants. A computer based image processing technique had been presented in this article addressing the current industry demand for an automated sorting system. Recycling plants are encouraged to utilize such technique in order to facilitate their quality assurance procedures. The system is capable of identifying 7 different materials in a received demolition batch for a high accuracy rate of $85 \%$. More investigations is required in order to develop a robotic claw to use the output data from the presented system which finalizes the sorting process.

\section{Conflict of Interest}

The authors declare that the research was conducted in the absence of any commercial or financial relationships that could be construed as a potential conflict of interest.

\section{References}

[1] G. K. Ding, "Sustainable construction-The role of environmental assessment tools," Journal of environmental management, vol. 86, no. 3, pp. 451-464, 2008.

[2] G. K. Ding, "Developing a multicriteria approach for the measurement of sustainable performance," Building Research \& Information, vol. 33, no. 1, pp. 3-16, 2005.

[3] V. W. Tam, K. N. Le, and C. N. Tran, "Optimizing life-cycle carbon emissions for achieving concrete credits in Australia," in Proceedings of the 21st International Symposium on Advancement of Construction Management and Real Estate, 2018: Springer, pp. 1077-1087.

[4] V. W. Tam, K. N. Le, C. N. Tran, and I. C. S. Illankoon, "A review on international ecological legislation on energy consumption: greenhouse gas emission management," International Journal of Construction Management, pp. 1-12, 2019.

[5] V. W. Tam, K. N. Le, C. N. Tran, and I. C. S. Illankoon, "An Exploratory Model on GreenhouseGas Emissions and Costing Assessment in Building Operation Stage," in Conference on Innovative Production and Construction 2017 (Perth), 2017: World Scientific.

[6] V. W. Tam, K. N. Le, C. N. Tran, X. Wang, and J. Wang, "A review of international green building designs," International Journal of Construction Project Management, vol. 9, no. 1, pp. 3-18, 2017.

[7] A. Akbarnezhad, K. C. G. Ong, M. H. Zhang, and C. T. Tam, "Acid Treatment Technique for Determining the Mortar Content of Recycled Concrete Aggregates," Journal of Testing and Evaluation, vol. 41, 2013.

[8] L. Evangelista and J. de Brito, "Mechanical behaviour of concrete made with fine recycled concrete aggregates," Cement and concrete composites, vol. 29, no. 5, pp. 397-401, 2007.

[9] F. Sartipi and E. Zarqam, "Recycled concrete and the advantages of using recycled aggregates," presented at the 3rd International congress on architecture, civil engineering and urban development, Tehran, 2016.

[10] V. W. Y. Tam, M. Soomro, and A. C. J. Evangelista, "A review of recycled aggregate in concrete applications," (in English), Construction and Building Materials, 2018.

[11] T. Boulos, F. Sartipi, and K. Khoshaba, "Bibliometric analysis on the status quo of robotics in construction," Journal of Construction Materials, vol. 1, pp. 2-3, 2020. 
[12] A. Todhunter, M. Crowley, M. Gholamisheverini, and F. Sartipi, "Advanced technological implementation of construction and demolition waste recycling," Journal of Construction Materials, vol. 1, no. 1, 2019, doi: https://doi.org/10.36756/JCM.v1.1.3.

[13] G. E. Metcalf and D. Weisbach, "The design of a carbon tax," Harv. Envtl. L. Rev., vol. 33, p. 499, 2009.

[14] D. Pearce, "The role of carbon taxes in adjusting to global warming," The economic journal, vol. 101, no. 407, pp. 938-948, 1991.

[15] D. Barker, S. Turner, P. Napier-Moore, M. Clark, and J. Davison, "CO2 capture in the cement industry," Energy procedia, vol. 1, no. 1, pp. 87-94, 2009.

[16] K. Vatopoulos and E. Tzimas, "Assessment of CO2 capture technologies in cement manufacturing process," Journal of cleaner production, vol. 32, pp. 251-261, 2012.

[17] A. Gharizadeh, F. Sartipi, E. Ayoubi, and A. Severino, "The chemical reactor design configuration of $\mathrm{CO} 2$ concrete green solution," Journal of Construction Materials, vol. 1, pp. 25, 2020, doi: http://doi.org/10.36756/JCM.v1.2.5.

[18] A. Todhunter, M. Crowley, F. Sartipi, and K. Jegendran, "Use of the by-products of postcombustion carbon capture in concrete production: Australian case study," Journal of Construction Materials, vol. 1, no. 1, pp. 1-1, 2019, doi: https://doi.org/10.36756/JCM.v1.1.1.

[19] M. Gamil, A. Gharizadeh, and F. Sartipi, "A review on graphene reinforced cement composite: technical approach for ecofriendly construction," Journal of Construction Materials, vol. 1, no. 1, 2019, doi: https://doi.org/10.36756/JCM.v1.1.4. Institute of Construction Materials.

[20] F. Sartipi, A. Gharizadeh, and M. Gamil, "Electrical resistance of graphene reinforced cement paste," Journal of Construction Materials, vol. 1, no. 1, 2019, doi:

https://doi.org/10.36756/JCM.v1.1.5. Institute of Construction Materials.

[21] F. Hollstein, Í. Cacho, S. Arnaiz, and M. Wohllebe, "Challenges in automatic sorting of construction and demolition waste by hyperspectral imaging," in Advanced Environmental, Chemical, and Biological Sensing Technologies XIII, 2016, vol. 9862: International Society for Optics and Photonics, p. 98620J.

[22] K. Anding, E. Linß, H. Träger, M. Rückwardt, and A. Göpfert, "Optical identification of construction and demolition waste by using image processing and machine learning methods," in 14th Joint Int. IMEKO Symp., Jena, Germany, 2011.

[23] F. Sartipi, "Organizational structure of construction entities based on the cooperative game theory," Journal of Construction Materials, vol. 1, no. 2, 2020, doi: https://doi.org/10.36756/JCM.v1.2.1. Institute of Construction Materials.

[24] A. Todhunter, M. Crowley, and F. Sartipi, "Construction productivity indices in socialism compared with capitalism," Journal of Construction Materials, vol. 1, no. 1, 2019, doi: https://doi.org/10.36756/JCM.v1.1.2.

[25] J. Luliano, A. Singh, and F. Sartipi, "Political-economical evaluation of CO2 capture in Australian building sector," Journal of Construction Materials, vol. 1, pp. 3-2, 2020, doi: http://doi.org/10.36756/JCM.v1.3.2. 